\title{
Subject Visit Start Date Time
}

National Cancer Institute

\section{Source}

National Cancer Institute. Subject Visit Start Date Time. NCI Thesaurus. Code C83436.

The date and time a subject visit began. 